\title{
Evolution of Quantum State in the Mesoscopic LC Circuit Driven by a Time Dependent Source
}

\author{
Xiaojian Xia \\ Department of Physics, Quanzhou Normal College \\ Quanzhou 36200 Fujian China
}

Tel: 86-130-459-50887_E-mail: xiaxiaojian18@eyou.com

Received: January 4, $2011 \quad$ Accepted: January 19, $2011 \quad$ Published: February 1, 2012
doi:10.5539/apr.v4n1p241
URL: http://dx.doi.org/10.5539/apr.v4n1p241

\begin{abstract}
We obtained the exact solution of time evolution by applying the Wei-Norman method.The quasi-probability distribution function of phase space is used to investigate the quantum state of the mesoscopic LC circuit in coherent state. The results show that the function of the system is the two dimensional motional gauss wave-packet. The centre charge and flux of the wave-packet vary with cosine and sine wave.
\end{abstract}

Keywords: LC circuit, Mesoscopic circuit, Coherent states, Evolution of quantum state, The quasi-probability distribution functions of phase space

\section{Introduction}

With the rapid development of nano technology, the miniaturization of circuits in electric devices and the trend of intense integration became more and more remarkable. The integration has achieved the scale of atomic inch recently. When the circuits scale have decreased to the scale of electric elastic collision. We must consider the effect of device and circuit. Since seventy years of twenty century, Louisell first discuss quantum fluctuation, Cheng-bin ,Cui yuan-shun and Wang ji-suo have investigated the quantum effect and effects in Coulomb blockade in mesoscopic LC and RLC circuit. In literature (Cheng, Li, et al., 1997), the conclusion that the amplitude of voltage in the circuit must be quantized is obtained. JI ying-hua has investigated the effect of mesoscopic LC and RLC circuit under pulse signal. Zhou xiao-fang has investigated the evolution of quantum state driven by a time dependent source with gauge transform of algebra dynamic method. In this paper, starting from the classic motion of LC circuit, we use the Wei-Norman method to obtain the time operator driven by a time dependent source.The result is the same as in the literature (Zhou, 2007). We use the quasi-probability distribution to investigate the mesoscopic LC circuit.The results show that the wigner function is two dimensional gauss wave-packet. The centre of charge and flux of the wave-packet vary with cosine and sine wave.

\section{The Time Operator of Quantum State in Mesoscopic LC Circuit with Dependent Sources}

In the macroscopic lumped parameter LC series circuit, we assume that when $\mathrm{t}<0$, there is no voltage source acting on circuit and the circuit is stable. From time $\mathrm{t}=0$, there is a voltage signal sources $e(t)=\varepsilon(t) u(t)$ acting on the circuit. $u(t)$ is step function. According to the Kirchhof 's voltage law, the equation is

$$
L \ddot{q}+L \omega^{2} q=e(t)
$$

Where $\omega^{2} L C=1$, and $\mathrm{q}$ is the capacitance charge, $p=L \dot{q}$ is the flux of the circuit. The correspond- ance to equation (1) of quantization Hamilton operator in LC Series Circuit is

$$
\hat{H}=\frac{1}{2 L} \hat{p}^{2}+\frac{1}{2} L \omega^{2} \hat{q}^{2}-\hat{q} e(t)
$$

$\hat{q}$ and $\hat{p}$ is charge operator and flux operator with the relation $\hat{p}=L \dot{\hat{q}}$ and $[\hat{q}, \hat{p}]=i \hbar$. We introduce $\hat{a}$ as lowering operator and $\hat{a}^{+}$as raising operator, and make the following expressing 


$$
\hat{a}^{+}=\frac{\alpha}{\sqrt{2}}\left(\hat{q}+i \frac{1}{\omega L} \hat{p}\right) \quad \hat{a}=\frac{\alpha}{\sqrt{2}}\left(\hat{q}-i \frac{1}{\omega L} \hat{p}\right)
$$

Where $\alpha=\sqrt{\frac{L \omega}{\hbar}}$, then Hamilton (2) can be expressed as

$$
\hat{H}=\hbar \omega\left(\hat{N}+\frac{1}{2}\right)-e(t) \frac{1}{\alpha \sqrt{2}}\left(\hat{a}^{+}+\hat{a}\right)=\hat{H}_{0}+V(t)
$$

Where $\hat{H}_{0}=\hbar \omega\left(\hat{N}+\frac{1}{2}\right), \hat{N}=\hat{a}^{+} \hat{a}, V(t)=-e(t) \frac{1}{\alpha \sqrt{2}}\left(\hat{a}^{+}+\hat{a}\right)$, we can express the Hamilton in the interaction representation as

$$
\hat{H}_{I}=e^{\frac{i \hat{H}_{0} t}{\hbar}} V(t) e^{-\frac{\hat{H}_{0} t}{\hbar}}=-e(t) \frac{1}{\alpha \sqrt{2}}\left(\hat{a}^{+} e^{i \omega t}+\hat{a} e^{-i \omega t}\right)=-\left(F(t) \hat{a}^{+}+F^{*}(t) \hat{a}\right)
$$

Where $_{F(t)}=e(t) \frac{1}{\alpha \sqrt{2}} e^{i \omega t}$, in the interaction representation, from the schrodingor equation, the evolution of time operator is obtained as

$$
\left[i \hbar \frac{\partial}{\partial t} \hat{U}_{I}\right] \hat{U}_{I}^{-1}(t)=\hat{H}_{I}
$$

We can slove the schrodinger equation with Hamilton (4) using Wei-Norman method. We assume the solution as

$$
\hat{U}_{I}(t)=e^{C(t)} e^{Z(t) \hat{a}^{+}} e^{B(t) \hat{a}}
$$

Substituting the expression (7) into (6), making use of operator derivation rule and Baker-Haus dorff formula. We obtain the coefficients satisfying the equations

$$
\dot{C}(t)-\dot{B}(t) z(t)=0 \quad i \hbar \dot{Z}(t)=F(t) \quad i \hbar \dot{B}(t)=F^{*}(t)
$$

Then we have

$$
C(t)=-\int_{0}^{t} Z(\xi)\left[Z^{\prime}(\xi)\right]^{*} d \xi \quad Z(t)=-B^{*}(t)=\frac{1}{i \hbar} \int_{0}^{t} F\left(t^{\prime}\right) d t^{\prime}
$$

We obtain the time operator of evolution from the interaction representation backing to the original representation.

$$
\hat{U}(t)=e^{-\frac{i \hat{H}_{0} t}{\hbar}} U_{I}(t)=e^{-i \omega\left(\hat{N}+\frac{1}{2}\right) t} U_{I}(t)=e^{i \operatorname{Im} C(t)} e^{-i \omega\left(\hat{N}+\frac{1}{2}\right) t} e^{\left[Z(t) \hat{a}^{+}-Z^{*}(t) \hat{a}\right]}
$$

From formula (10),we can see it is the same as that in literature (Zhou, 2007). It is easy to test the time operator to be unitary and the time operator satisfy $\hat{U}^{+}(t) \hat{U}(t)=1$.

\section{The Quasi-probability Distribution Function of Mesoscopic LC Circuit-wigner Function}

We use $|n\rangle$ to represent the eigenstate of eigenvalue $\mathrm{n}$. As the voltage sources act on the system from $\mathrm{t}=0$. Before $\mathrm{t}=0$ the system is stable. We consider the initial quantum state of the system as $\left|\psi\left(0_{-}\right)\right\rangle=|0\rangle$. From equation (10), we obtain the quantum state at any time as

$$
|\psi(t)\rangle=e^{i \operatorname{Im} C(t)} e^{-i \omega t\left(\hat{N}+\frac{1}{2}\right)}|Z\rangle
$$

Using $e^{z \hat{a}^{+}-z^{*} \hat{a}}=e^{-\frac{1}{2} z^{*} z} e^{z \hat{a}^{+}} e^{-z^{*} \hat{a}}$, then we have

$$
\begin{gathered}
\left.|z\rangle=e^{-\frac{1}{2} z^{*} z} \sum_{0}^{\infty} \frac{z^{n}}{\sqrt{n !}} n\right\rangle \\
\langle q \mid \psi(t)\rangle=e^{i \operatorname{Im} C(t)} e^{-\frac{1}{2} z^{*} z} \sum_{n=0}^{\infty} \frac{z^{n}}{\sqrt{n !}} e^{-i \omega t\left(n+\frac{1}{2}\right)}\langle q \mid n\rangle
\end{gathered}
$$

Because 


$$
\langle q \mid n\rangle=\left(\frac{\alpha}{\sqrt{\pi} 2^{n} n !}\right)^{\frac{1}{2}} e^{-\frac{1}{2} \alpha^{2} q^{2}} H_{n}(\alpha q)
$$

Then we have

$$
\langle q \mid \psi(t)\rangle=\left(\frac{\alpha}{\sqrt{\pi}}\right)^{\frac{1}{2}} e^{-i\left(\frac{1}{2} \omega t-\operatorname{Im} C(t)\right)} e^{-\frac{1}{2} z^{*} z-\frac{1}{2} \alpha^{2} q^{2}} \sum_{n=0}^{\infty} \frac{1}{n !}\left(\frac{z e^{-i \omega t}}{\sqrt{2}}\right)^{n} H_{n}(\alpha q)
$$

Because

$$
\sum_{n=0}^{\infty} \frac{1}{n !} \eta^{n} H_{n}(\xi)=e^{-\eta^{2}+2 \eta \xi}
$$

Then we have

$$
\begin{gathered}
\langle q \mid \psi(t)\rangle=\left(\frac{\alpha}{\sqrt{\pi}}\right)^{\frac{1}{2}} e^{-i\left(\frac{1}{2} \omega t-\operatorname{Im} C(t)\right)} e^{-\frac{1}{2} z^{*} z-\frac{1}{2} \alpha^{2} q^{2}} \exp \left[-\left(\frac{z e^{-i \omega t}}{\sqrt{2}}\right)^{2}+2\left(\frac{z e^{-i \omega t}}{\sqrt{2}}\right) \alpha q\right] \\
\left\langle q+\frac{1}{2} x \mid \psi(t)\right\rangle=\left(\frac{\alpha}{\sqrt{\pi}}\right)^{1 / 2} \exp \left\{-i\left[\frac{1}{2} \omega t-\operatorname{Im} C(t)-\frac{1}{2}|z|^{2} \sin 2(\omega t-\arg z)\right]+\sqrt{2} \alpha\left(q+\frac{1}{2} x\right)|z| \sin (\omega t-\arg z)\right\} \\
\exp \left\{-\frac{1}{2} \alpha^{2}\left[q+\frac{1}{2} x-\frac{\sqrt{2}}{2} \mid z \cos (\omega t-\arg z)\right]^{2}\right\} \\
\left\langle\psi(x) \mid q-\frac{1}{2} x\right\rangle=\left(\frac{\alpha}{\sqrt{\pi}}\right)^{1 / 2} \exp \left\{\left[\frac{1}{2} \omega t-\operatorname{Im} C(t)-\frac{1}{2}|z|^{2} \sin 2(\omega t-\arg z)\right]+\sqrt{2} \alpha\left(q-\frac{1}{2} x\right)|z| \sin (\omega t-\arg z)\right\} \\
\exp \left\{-\frac{1}{2} \alpha^{2}\left[q-\frac{1}{2} x-\frac{\sqrt{2}}{2}|z \cos (\omega t-\arg z)|\right]^{2}\right\}
\end{gathered}
$$

In 1932, wigner first introduce quasi-probability distribution functions in phase space-wigner function, using coordinate and momentum as variables. There is a corresponding relation between the quantum state and the wigner function. And the quantum state is determined. We use the definition in literature (Zhou, 2007).

$$
\begin{aligned}
W(p, q, t)= & \left.\frac{1}{2 \pi \hbar} \int_{-\infty}^{+\infty}\left|q+\frac{1}{2} x\right| \psi(t)\right\rangle\left\langle\psi(t) \mid q-\frac{1}{2} x\right\rangle e^{i p x / \hbar} d x \\
= & \frac{1}{2 \pi \hbar} \frac{\alpha}{\sqrt{\pi}} \exp \left\{\alpha^{2}\left[q-\frac{\sqrt{2}}{\alpha}|z| \cos (\alpha t-\arg z)\right]^{2}\right\} \times \\
& \int_{-\infty}^{+\infty} \exp \left\{\alpha^{2}\left[q-\frac{\sqrt{2}}{\alpha}|z| \cos (\omega t-\arg z)\right]^{2}\right\} \times \int_{-\infty}^{+\infty} \exp \left[-\frac{1}{4} \alpha^{2} x^{2}+\frac{i p x}{\hbar}-i \sqrt{2} \operatorname{ax}|z| \sin (\omega t-\arg z)\right] d x \\
= & \frac{1}{\pi \hbar} \exp \left\{\alpha^{2}\left[q-\frac{\sqrt{2}}{\alpha}|z| \cos (\omega t-\arg z)\right]^{2}\right\} \times \exp \left\{\frac{1}{\alpha^{2} \hbar^{2}}[p-\sqrt{2} \alpha \hbar|z| \sin (\omega t-\arg z)]^{2}\right\}
\end{aligned}
$$

We use the following integral formula [14]

$$
\int_{-\infty}^{+\infty} e^{-q^{2} x^{2}} \sin [p(x+\lambda)] d x=\frac{\sqrt{\pi}}{q} e^{-\frac{p^{2}}{4 q^{2}}} \sin (p \lambda) \int_{-\infty}^{+\infty} e^{-q^{2} x^{2}} \cos [p(x+\lambda)] d x=\frac{\sqrt{\pi}}{q} e^{-\frac{p^{2}}{4 q^{2}}} \cos (p \lambda)
$$

From equation(20), we can see the formation in the phase space is two dimensional gauss wave-packet when mesoscopic LC circuit is in coherent state.

\section{The Coherent State in Fock Presentation is the Gauss Wave-packet in Coordinate Presentation}

From equation (12), we get

$$
\langle q \mid z\rangle=e^{-\frac{1}{2} z^{*} z} \sum_{0}^{\infty} \frac{z^{n}}{\sqrt{n !}}\langle q \mid n\rangle
$$

We substitute (14) into (22)and employ the relation (16), then we get the following expression 


$$
\psi_{q}(q)=\langle q \mid z\rangle=C\left(\frac{m \omega}{\pi \hbar}\right)^{1 / 4} e^{-\frac{1}{2} z^{*} z} e^{-z^{2}} e^{-\frac{1}{2}(\xi-2 z)^{2}}
$$

Where $C=1+\sqrt{2}, \xi=q \sqrt{m \omega / \hbar}$. The equation(23)shows that the coherent state in fock presentation is the gauss wave-packet in the coordinate presentation. The coherent state is obtained when the time operator expressed in (10) acting on the vacuum state, and remains coherent state under free harmonic oscillator operator.

\section{Results and Discussions}

We use Wei-Norman method to obtain the exact time operator of mesoscopic LC circuit driven by dependent source. The results show that if the initial state is vacuum state, then the state is coherent state at anytime. We use the quasi-probability distribution to investigate the mesoscopic LC circuit.The results show that the wigner function is two dimensional gauss wave-packet. The centre of charge and flux of the wave-packet vary with cosine and sine wave.

\section{References}

Cheng B, Li Y Q, et al. (1997). Quantum effects of charge in the mesoscopic circuit. Acta Physica Sinica, 46(1): 131-133 (in Chinese).

Cui Y S, \& Bian Q P. (1999). The influence of source on quantum fluctuation in an active mesoscopic circuit. College Physics, 18(4): 10-12 (in Chinese).

Cui Y S. (1998). Quantum fluctuations of voltage and current in mesoscopic LC circuit. Acta Photonica Sinica, 27(6), 517-520 (in Chinese).

Cui Y S. (1999). Quantum fluctuations under squeezed vacuum state and Influence of source on the fluctuation in a mesoscopic coupled circuit. Chinese Journal of computational physics, 16(3): 321-327. (in Chinese).

I. S. G., \& L. M. R. (2004). Table of Integrals series and products, sixth edition. Elsevier(Singapore) Pte.Ltd.

Ji Y H, Luo h M, et al. (2004). Preparation of Schrodinger cat state via a mesoscopic LC circuit. Acta Physica Sinica, 53(8): 2534-2538 (in Chinese).

Ji Y H, Rao J P, et al. (2002). Quantum tunneling effect in the mesoscopic LC circuit. Acta. Phys.Sinica. 51(2), 395-398. (in Chinese).

Louisell W H. (1973). Quantum Statistical Properties of Radiation. New York John wiley,1973. Chapter.

Sun C P, \& Xiao Q, Wei. (1991). Norman algebraic method solving the evolution of the coherent states of electron in two dimensions. Commun. Theor. Phys., 16(3):359-362.

Wang C, Zhang Li, et al. (1997). Classical and Quantum detuning. College Physics, 16(3):7-10. (in Chinese).

Wang J S, Liu T K, et al. (2000a). Quantum fluctuations of a mesoscopic capacitance coupling circuits in a displaced squeezed fock state. Acta. Phys. Sinica, 49(11) 2271-2275. (in Chinese).

Wang J S, Liu T K, et al. (2000b). Quantum effects of a non-dissipatived mesoscopic circuit with inductance coupling. Acta Photonica Sinica, 29(1): 22-26 (in Chinese).

Wigner E. (1932). On the quantum correction for thermodynamic equilibrium. Phys. Rev., 40:749-759.

Zhou X F. (2007). Evolution of quantum state in the mesoscopic LC circuit driven by time dependent source. Chinese journal of quantum electronics, 24(5): 600-604 (in Chinese). 